\title{
Inhaltsverzeichnis
}

1 Rettungsdienst, Berufsbild, Recht

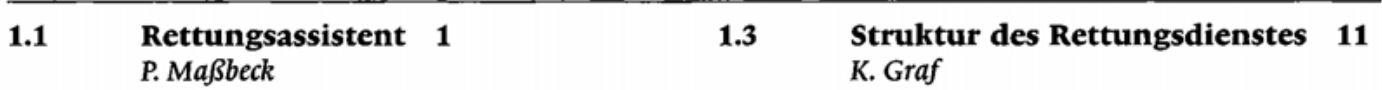

1.2 Recht im Rettungsdienst (RD) 8

J. Möller

2 Medizinische Grundlage: Funktionelle Anatomie, Zytologie

2.1 Körper: Bau und Funktion im $\quad 2.1 .7$ Urogenitalorgane 41

Überblick 13

2.1.8 Haut, Hautanhangsgebilde 45

E. Häusler

2.1.9 Sinnesorgane 46

2.1.1 Atmungssystem 13

2.1.10 Nervensystem 49

2.1.2 Blutkreislauf 19

2.1.11 Regulationssysteme 57

2.1.3 Blut 25

2.1.4 Lymphatisches System 28

2.1.5 Stütz- und Bewegungsapparat: $\quad$ E. Häusler

$2.2 \quad$ Zelle $\quad 57$

$\begin{array}{lllll} & \text { Knochen, Gelenk, Muskel } & 28 & 2.2 .1 & \text { Bau } 59 \\ \text { 2.1.6 } & \text { Verdauungsorgane } 35 & & 2.2 .2 & \text { Stoffwechsel } 61\end{array}$

3 Naturwissenschaftliche Grundlagen: Chemie, Physik

\begin{tabular}{lll}
\hline 3.1 Chemie 63 & 3.2 & $\begin{array}{l}\text { Physik 73 } \\
\text { B.Steinbach }\end{array}$
\end{tabular}

3.1.1 Grundbegriffe 63

3.2.1 Grundbegriffe, Größen 73

3.1.2 Anorganische Chemie 64

3.2.2 Optik 75

3.1.3 Organische Chemie 68

3.2.3 Elektrizität 77

$4 \quad$ Hygiene im Rettungsdienst

\begin{tabular}{lllll}
\hline 4.1 & $\begin{array}{l}\text { Allgemeine Infektionslehre } \\
\text { G. Lietz }\end{array}$ & 79 & 4.3 & $\begin{array}{l}\text { Krankenhausinfektion } \\
\text { G. Lietz }\end{array}$
\end{tabular}

4.2 Hygienemaßnahme: Desinfektion, $4.4 \quad$ Schutzimpfung 84 Sterilisation $\mathbf{8 1}$ G. Lietz

G. Lietz

$5 \quad$ Allgemeine Notfallmedizin

\begin{tabular}{|c|c|c|c|}
\hline 5.1 & $\begin{array}{l}\text { Einsatzart, Einsatzablauf } 87 \\
\text { K. Luszeit }\end{array}$ & 5.2 & $\begin{array}{l}\text { Vitlalfunktion } \\
\text { K. Jentsch }\end{array}$ \\
\hline 5.1 .1 & $\begin{array}{l}\text { Einsatzart, Stichwörter zur } \\
\text { Alarmierung } 87\end{array}$ & $\begin{array}{l}5.2 .1 \\
5.2 .2\end{array}$ & $\begin{array}{l}\text { Störungen } 89 \\
\text { Kontrolle } 89\end{array}$ \\
\hline
\end{tabular}




\subsection{Sofortmaßnahmen am Notfallort 90 \\ R. Sick}

5.3.1 Bewußtseinsstörung 90

5.3.2 Störung der Atmung 93

5.3.3 Herz-Kreislauf-Stillstand 99

5.3.4 Schock 99

5.3.4.1 Bedrohliche Blutung 100

$5.4 \quad$ Lagerung 103

R. Sick

5.5 Erweiterte Maßnahme: Intubation

(Definition, Indikation,

Material) 105

K. Jentsch

$5.6 \quad$ Beatmung, Absaugung 112

K. Jentsch

5.6.1 Beatmung: Definition, Indikation 112

5.6.2 Absaugung 112

5.6.3 Beatmungsform 113

5.6.4 Komplikation, Gefahr, Nachsorge 115
5.8

5.9

5.9 .1

5.9 .2

5.9 .3

5.10

5.10 .1

5.10 .2

\section{Blutdruckmessung 115} K. Jentsch

\author{
Herz-Lungen-Wiederbelebung \\ (HLW) 117 \\ K. Jentsch
}

Verbandstechnik, Ruhigstellung 119 K. Jentsch

\section{Wunde 119}

Fraktur 120

Material, Technik 121

Patientenbetreuung, Krisenintervention 121 B. Coellen

Patientenbetreuung im Alltag, Großschadensfall 121

Belastungsfaktoren bei Krisenintervention 123

\section{Medizinische Fachrichtungen}

\begin{tabular}{|c|c|c|c|}
\hline 6.1 & Innere Medizin 127 & 6.2 .3 & Abdominalchirurgie 144 \\
\hline & F. Bertschat & 6.2.3.1 & Ösophagus (Speiseröhre) 145 \\
\hline 6.1 .1 & Vom Symptom zur Diagnose 127 & 6.2.3.2 & Magen 145 \\
\hline 6.1 .2 & Von der Diagnose zur Therapie 127 & 6.2.3.3 & Dünndarm 146 \\
\hline 6.1 .3 & Teilgebiete 128 & 6.2.3.4 & Appendix vermiformis (Wurmfortsatz \\
\hline 6.1.3.1 & Angiologie 128 & & des Bliddarmes) 147 \\
\hline 6.1.3.2 & Endokrinologie 129 & 6.2.3.5 & Dickdarm (Kolon, Rektum), \\
\hline 6.1.3.3 & Gastroenterologie 129 & & Analkanal 147 \\
\hline 6.1.3.4 & Hämatologie, Onkologie & 6.2.3.6 & Leber 149 \\
\hline 6.1.3.5 & Kardiologie 135 & 6.2.3.7 & Gallenblase, Gallenwege 149 \\
\hline 6.1.3.6 & Nephrologie 137 & 6.2.3.8 & Pankreas (Bauchspeicheldrüse), Milz \\
\hline 6.1.3.7 & $\begin{array}{l}\text { Pneumologie: Lungen-, } \\
\text { Bronchialheilkunde } 139\end{array}$ & 6.2 .4 & $\begin{array}{l}\text { (Lien) } \quad 149 \\
\text { Gefäßchirurgie } \quad 150\end{array}$ \\
\hline 6.1.3.8 & Rheumatologie 140 & 6.2 .5 & $\begin{array}{l}\text { Unfallchirurgie: allgemeine } \\
\text { Frakturenlehre } 151\end{array}$ \\
\hline 6.2 & $\begin{array}{l}\text { Chirurgie } 140 \\
\text { U. Finke }\end{array}$ & 6.3 & Geburtshilfe, Gynäkologie 153 \\
\hline 6.2 .1 & Allgemeinchirurgie 141 & & \\
\hline 6.2.1.1 & Chirurgischer Eingriff 141 & 6.3 .1 & Schwangerschaft 153 \\
\hline 6.2.1.2 & Wunde, Wundheilung, -behand- & 6.3 .2 & Gestörte Schwangerschaft 154 \\
\hline & lung 141 & 6.3 .3 & Geburt, Geburtsüberwachung 155 \\
\hline 6.2.1.3 & Verbandlehre 142 & 6.3 .4 & Regelwidrige Geburt 157 \\
\hline 6.2 .2 & Thoraxchirurgie 143 & 6.3.4.1 & Geburtsdauer 157 \\
\hline 6.2.2.1 & Mammakarzinom (Brustkrebs) 143 & 6.3.4.2 & Vaginale Operation, Kaiserschnitt \\
\hline 6.2.2.2 & Lungen, Pleura 144 & 6.3.4.3 & Beckenendlage, Zwillinge 158 \\
\hline
\end{tabular}


6.3.4.4 Nachgeburtsperiode 159

6.3.5 Wochenbett 159

\begin{tabular}{|c|c|}
\hline 6.4 & $\begin{array}{l}\text { Pädiatrie } 160 \\
\text { J. Sonntag }\end{array}$ \\
\hline 6.4 .1 & Wärmehaushalt \\
\hline 6.4 .2 & Atmung 160 \\
\hline 6.4 .3 & Herz-Kreislauf-System 161 \\
\hline 6.4 .4 & $\begin{array}{l}\text { Stoffwechsel, Wasser-, Elektrolyt- } \\
\text { Salzhaushalt } 162\end{array}$ \\
\hline 6.4 .5 & Medikation 162 \\
\hline 6.5 & $\begin{array}{l}\text { Anästhesie } 162 \\
\text { H. Moecke }\end{array}$ \\
\hline
\end{tabular}

6.5.1 Anästhesie, Analgesie, Sedierung im Rettungsdienst 162

6.5.2 Durchführung 164

6.6 Orthopädie 168 J. Grifka

6.6.1 Kopf, Hals: Zervikalsyndrom 168

6.6.2 Schulter, Arm 169

6.6.3 Brustwirbelsäule (BWS) 170

6.6.4 Lendenwirbelsäule (LWS) 170

6.6.5 Hüfte, Bein 171

6.7 Urologie 172

G. Haupt, J. Pannek, Th. Senge

6.7.1 Gutartige Krankheiten 172

6.7.2 Bösartige Krankheiten 173

\section{$6.8 \quad$ Neurologie 174}

Th. Lempert, W. Christe
6.8.1 Leitsymtome 174

6.8.1.1 Kopfschmerz 174

6.8.1.2 Akute Bewußtseinsstörung 175

6.8.1.3 Akute Lähmung 176

6.8.1.4 Anfall 176

$6.9 \quad$ Psychiatrie 177

S. Schütze

6.9.1 Psychose 177

6.9.2 Erlebnisreaktive Störung 178

6.9.2.1 Sucht 178

6.9.2.2 Neurotische Störung 179

6.9.3 Psychiatrische Arbeit 179

6.10 Hals-Nasen-Ohren-Heilkunde (HNO) 179

H. Hildmann

6.10.1 Ohr 180

6.10.2 Nase, Nasennebenhöhlen 182

6.10.3 Lymphatischer Rachenring: Gaumen, -Rachenmandel 183

6.10.4 Speicheldrüsen 184

6.10.5 Kehlkopf, Halsorgane 184

6.10.6 Stimme, Sprache, Gesichtschirurgie 186

6.11 Augenheilkunde 186 U. Gronemeyer

6.11.1 Lider, Tränenwege, Bindehaut 186

6.11.2 Hornhaut, Linse, Gefäßhaut 187

6.11.3 Netzhaut, Sehnerv 188

6.11.4 Grüner Star (Glaukom) 188

\section{$7 \quad$ Spezielle Notfallmedizin}

\begin{tabular}{|c|c|c|c|}
\hline 7.1 & $\begin{array}{l}\text { Akute Kerz-Kreislauf-Störung } \\
\text { Th. Störk }\end{array}$ & $\begin{array}{l}7.1 .5 .3 \\
7.1 .5 .4\end{array}$ & $\begin{array}{l}\text { Kardiogener Schock } 197 \\
\text { Anaphylaktischer Schock } 197\end{array}$ \\
\hline 7.1 .1 & Angina pectoris 189 & 7.1.5.5 & Septisch-toxischer Schock \\
\hline 7.1 .2 & $\begin{array}{l}\text { Akuter Myokardinfarkt (Herzinfarkt, } \\
\text {-muskelinfarkt) } 190\end{array}$ & 7.1.5.6 & $\begin{array}{l}\text { (Endotoxinschock) } 198 \\
\text { Weitere Schockformen } 198\end{array}$ \\
\hline 7.1 .3 & Herzinsuffizienz 192 & 7.1.6 & Synkope 198 \\
\hline 7.1 .3 .1 & $\begin{array}{l}\text { Kardiales Lungenödem } \\
\text { (Rückwärtsversagen) } 193\end{array}$ & $\begin{array}{l}7.1 .7 \\
7.1 .8\end{array}$ & $\begin{array}{l}\text { Hypertensive Krise } 199 \\
\text { Lungenembolie } 199\end{array}$ \\
\hline 7.1.3.2 & $\begin{array}{l}\text { Kardiogener Schock: } \\
\text { Pumpversagen } 193\end{array}$ & 7.2 & Akute Störung der Atmung 200 \\
\hline 7.1.4 & $\begin{array}{l}\text { Herzrhythmusstörung, EKG, } \\
\text { Herzschrittmacher } 193\end{array}$ & 7.2 .1 & Ing der Atemwege: \\
\hline 7.1.5 & Schock 195 & & Bolusobstruktion 201 \\
\hline 7.1.5.1 & Schockzeichen, Erstmaßnahmen & 7.2 .2 & Aspiration 202 \\
\hline 7.1 .5 .2 & Volumenmangelschock 196 & 7.2 .3 & Asthma bronchiale \\
\hline
\end{tabular}


7.2.4 Pleura- und Thoraxverletzung 203

7.2 .5

7.2 .6

7.2.7

7.3 Verletzung des Bewegungsapparates 206 A. Tempka

7.3.1 Verletzungsarten 206

7.3.2 Erstbehandlung 208

7.4 Polytrauma: Erstversorgung am Unfallort 212

A. Tempka

7.4.1 Sofortmaßnahmen 213

7.4.2 Häufige Einzelverletzungen 213

7.5 Schädel-Hirn-Trauma (SHT) 215

Ch. K. Lackner, J. H. Widmann,

L. Schweiberer

7.5.1 Einteilung, Pathophysiologie 215

7.5.2 Notfalldiagnostik, Therapie 217

7.6 Verbrennung, Stromverletzung $\mathbf{2 2 2}$ K.-P. Wresch

7.6.1 Verbrennung 222

7.6.2 Strom- und Blitzunfall 227

7.7 Abdominaltrauma 229 D. Richter, A. Ekkernkamp

7.7.1 Stumpfes Bauchtrauma 229

7.7.2 Perforierende Bauchverletzung 230

7.7.3 Akutes Abdomen 231

7.8 Herz-Kreislauf-Stillstand, Kardiopulmonale Reanimation (CPR) 233 K. Jentsch

7.8.1 Herz-Kreilauf-Stillstand (HKS) 233

7.8.2 Kardiopulmonale Reanimation, Erwachsene 233

7.8.3 Reanimation von Säuglingen, Kleinkindern 236

7.9 Unterkühlung, Erfrierung 237 Th. Dietz

7.9.1 Unterkühlung 237

7.9.2 Erfrierung 238 $\mathbf{7 . 1 0}$ Intoxikation (Vergiftung) 239 C. Köppel

7.10.1 Art, Diagnose 239

7.10.2 Therapie 240

7.11 Akute Störung von WasserElektrolyt-, Säure-BasenHaushalt 243 Th. Dietz

7.11.1 Wasser- und Elektrolyt-Haushalt 243

7.11.1.1 Hypovolämie 244

7.11.1.2 Hypervolämie 244

7.11.2 Störung im Säure-Basen-Haushalt 245

7.11.2.1 Respiratorische Alkalos: Hyperventialtionssyndrom 245

7.11.2.2 Respiratorische Azidose 246

7.12 Akute Stoffwechselstörung 246 Th. Schirop

7.12.1 Zuckerkrankheit: Zuckerkoma, Zuckerschock 246

7.12.1.1 Coma diabeticum 246

7.12.1.2 Hypoglykämischer Schock 248

7.12.2 Coma uraemicum 249

7.12.2.1 Akutes Nierenversagen (ANV) 249

7.12.2.2 Chronisches Nierenversagen 250

7.12.3 Coma hepaticum 250

7.13 Gynäkologisch-geburtshilflicher Notfall 251

W. Friedmann, K. W. Dudenhausen

7.13.1 Geburtshilflicher Notfall 251

7.13.1.1 Placenta praevia 251

7.13.1.2 Vorzeitige Plazentalösung 252

7.13.1.3 Präeklampsie, Eklampsie 253

7.13.1.4 Frühgeburt 253

7.13.1.5 Nabelschnur-, Armvorfall 254

7.13.1.6 Verschleppte Querlage, Fruchtwasserembolie 255

7.13.1.7 Abgebrochene Hausgeburt, Plazentalösungsstörung 255

7.13.1.8 Abort 256

7.13.1.9 Extrauterine Schwangerschaft (EU) 256

7.13.2 Akutes Abdomen 257

7.13.3 Blutung 258

7.14 Pädiatrischer Notfall 258 J. Sonntag

7.14.1 Reanimation 258 
7.14.2 Früh- und

Neugeborenenversorgung 262

7.14.3 Plötzlicher Kindstod, Atemstörung, Herzkrankheit, Krampfanfall 264

7.14.4 Infektiös-toxische Krankheit 268

7.14.5 Vergiftung, Unfall 268

\subsection{Schlaganfall, Epileptischer}

Anfall 271

W. Christe, T. Lempert

7.15.1 Schlaganfall (Apoplexia cerebri) 271

7.15.2 Epileptischer Anfall, Epilepsie 273

\subsection{Psychischer}

Ausnahmezustand 275

S. Schütze

7.16.1 Organisch bedingter Notfall: akute

Verwirrtheit, Entzugsyndrom,

Intoxikation 275

7.16.2 Psychotische Erregung,

Suizidalität 276

7.16.3 Psychogene Krise: Angst, Panik 277

7.17 Hals-Nasen-Ohren-Notfall 277 H. Hildmann

7.17.1 Ohr: plötzlicher Hörverlust, Schwindel, Fazialislähmung 277

7.17.2 Nase 279

7.17.3 Gesichtsverletzung 279
7.17.4 Mund, Rachen: Verletzung, Blutung 280

7.17.5 Kehlkopf, Luftröhre 280

7.18 Urologischer Notfall 281

J. Pannek, G. Haupt, T. Senge

7.18.1 Harnverhaltung (Ischurie) 281

7.18.2 Nierenkolik 283

7.18.3 Akuter Hoden 284

7.18.4 Paraphimose (Spanischer Kragen),

Priapismus 285

7.18.5 Hämaturie (Blut im Urin) 286

7.18.6 Harnröhrenverletzung 286

7.19 Ophthalmologischer Notfall 286

$$
\text { U. Gronemeyer }
$$

7.19.1 Augenverletzung 286

7.19.1.1 Kontusion, Perforation 286

7.19.1.2 Verätzung, Verbrennung, Strahlenunfall, extraokuläres Trauma 287

7.19.2 Akute Augenkrankheit 288

7.20

Strahlen-, Chemieunfall 290

C. Köppel

7.20.1 Strahlenunfall 290

7.20.1.1 Strahlenwirkung 290

7.20.1.2 Unfallart, Selbstschutz 291

7.20.2 Chemieunfall 291

\section{Arzneimittel, Infusion, Sonde, Katheter, Drainage}

\begin{tabular}{llll}
\hline 8.1 Allgemeine Arzneimittellehre 293 & 8.2 .5 & $\begin{array}{l}\text { Calciumantagonist, Nitrat, } \\
\text { Detablocker 306 }\end{array}$
\end{tabular}

$\begin{array}{lll}\text { 8.1.1 Arznei-, Betäubungsmittel } 293 \\ \text { 8.1.2 Darreichungsformen 294 } \\ \text { 8.1.3 } & \text { Pharmokodynamik, -kinetik } 297\end{array}$

8.2 Notfallmedikamente 298 D. Ohlendorf

8.2.1 Analgetikum, Sedativum 299

8.2.2 Narkose: Injektionsnarkotikum 301

8.2.3 Psychopharmakum, Antihistaminikum 302

8.2.4 Hormone 303

8.2.4.1 Katecholamine: Adrenalin, Noradrenalin, Dopamin, Dobutamin 303

8.2.4.2 Insulin, Glukagon 305

8.2.4.3 Glukokortikoide 305
8.2.6 Antiarrhythmikum,

Antihypertensivum 308

8.2.7 Antiasthmatikum, Antiemetikum 308

8.2.8 Infusion 308

8.3 Infusions-, Injektionstechnik 309 St. Wallmeyer

8.3.1 Peripher-venöse Punktion 309

8.3.2 Zentralvenöse Punktion $\mathbf{3 1 1}$

8.3.3 Endobronchiale und intraossäre Applikation 312

8.4 Sonde, Katheter, Drainage $\mathbf{3 1 3}$ U. Beloch

8.4.1 Sonde, Katheter 313 
$\begin{array}{lllll}\text { 8.4.1.1 } & \text { Magen- und Duodenalsonde } & 313 & 8.4 .2 & \text { Drainage } 316 \\ \text { 8.4.1.2 } & \text { Harnblasenkatheter } 315 & & 8.4 .3 & \text { Pflege } 316\end{array}$

9 Geräte- und Fahrzeugkunde, Rettungs- und Löschtechnik

$\begin{array}{llll}9.1 & \begin{array}{l}\text { Medizinische Geräteverordnung } \\ \text { (MedGV), Medizinproduktegesetz }\end{array} & 9.7 & \begin{array}{l}\text { Rettungstechnik } \\ \text { R.-D. Erbe }\end{array}\end{array}$

(MPG) 319

Th. Schulz, I. Zydziak

9.2 Beatmungsgerät 319 R. Rossi

9.2.1 Beatmung des Notfallpatienten 321

9.2.2 Beatmungsüberwachung, -fehler 326

9.3 Pulsoxymetrie, Kapnometrie, -graphie 326

J. H. Widmann, Ch. K. Lackner,

St. Schmidbauer

9.3.1 Pulsoxymetrie 326

9.3.2 Kapnometrie, -graphie 327

9.4 Externe elektrische

Defibrillation 329

D. Maurer, Ph. Diehl

9.4.1 Defibrillation, Kardioversion 329

9.4.2 Geräte 330

9.5 Fahrzeugkunde,

Rettungsmittel 333

K. Luszeit

9.5.1 Landgebundenes Rettungsmittel (RM) 333

9.5.2 Luftrettungsmittel 334

9.5.3 Wasserrettungsfahrzeuge 334

9.5.4 Deutsche Industrienorm (DIN) für

Rettungsmittel 335

$9.6 \quad$ Luftrettung 336

K. Trottnow

9.6.1 Primärrettung, Sekundärtransport 336

9.6.2 Besatzung, Technik 337
9.7.1 Transporthilfsmittel 338

9.7.1.1 Rettungstrage 339

9.7.1.2 Rettung aus Höhe und Tiefe 340

9.7.2 Verkehrsunfall 342

9.7.2.1 Ausrüstung des Krankenwagens (KrKW) 342

9.7.2.2 Gefahren an der Unfallstelle 343

9.7.2.3 Einsatz einfacher Hilfsmittel 343

9.7.2.4 Retten von Personen aus PKW mit Airbag 346

9.7.2.5 Eingeklemmte Person, Sicherheitsgurt 349

9.7.3 Bahnbereich 350

9.7.3.1 Straßenbahn 350

9.7.3.2 S- und U-Bahn 350

9.7.3.3 Deutsche Bahn AG 351

9.7.4 Kraftbetriebene Maschinen 352

9.7.4.1 Rettung Eingeklemmter, hydrauliches Gerät 352

9.7.4.2 Hebegerät, Trennschleifer, Schneidbrenner 354

9.7.4.3 Atemschutzgerät, Sprungrettungsgerät 355

9.8 Löschtechnik 356

R. Erbe

9.8.1 Brandlehre 356

9.8.2 Löschmittel 357

9.8.3 Löschgerät 359

9.8.4 Löschtaktik 360

9.8.4.1 Grundsatz 360

9.8.4.2 Pulver-Feuerlöscher, elektrische Anlage, Löschdecke 361

9.8.4.3 Brandrauch, Fett-, elektrischer, Auto-, Gas-, Wohnungsbrand, brennende Person 361

10 Organisation, Einsatztaktik im Rettungsdienst

\begin{tabular}{llcll}
\hline 10.1 & $\begin{array}{l}\text { Rettungssysteme international: } \\
\text { USA, Österreich, Frankreich 365 }\end{array}$ & 10.2 & $\begin{array}{l}\text { Koordination } \quad 367 \\
\text { K. Luszeit }\end{array}$ \\
$\begin{array}{l}\text { St. Wallmeyer, W. Sladek, H. Michels, } \\
\text { B. Rösler, W. Antons }\end{array}$ & 10.2 .1 & Alarmierung, Einsatzablauf 367
\end{tabular}


10.2.2 Transportmittel, Einsatzkräfte 369

10.2.3 Fahrtaktik, Führungsaufgaben, Auslandseinsatz 369

10.3 Funk, Kommunikation 371 R. A. Müller

10.4 Fahrverhalten, Transporttechnik 375 M. Remy

10.4.1 Dringlicher Einsatz 375

10.4.2 Transport des Notfallpatienten 375

10.4.3 Übergabe im Krankenhaus 376

10.5 Patientenübergabe; Einsatzdokumentation, Qualitätsmanagement 377 H. Moecke

10.5.1 Patientenübernahme, -übergabe 377 10.5.2 Einsatzdokumentation 377

10.5.3 Qualitätsmanagement 378

10.6 Massenanfall von Verletzten 380 H. R. Paschen

10.6.1 Großschadensereignis 380
10.6.2 Sichtung 381

10.6.3 Schnell-Einsatz-Gruppen (SEG) 382

10.7 Gefahr an der Einsatzstelle $\mathbf{3 8 3}$ W. Reckert

10.7.1 Erkundung, Feststellung 383

10.7.2 Besondere Gefahr 383

10.7.2.1 Angstreaktion, Atemgift, Brandrauch 383

10.7.2.2 Atomare Gefahr, radioaktiver Stoff 385

10.7.2.3 Brand- und Schadstoffausbreitung 385

10.7.2.4 Chemischer Stoff 386

10.7.2.5 Einstürzendes Gebäudeteil 386

10.7.2.6 Explosion, Stichflamme, Druckgefäßzerknall 387

10.7.2.7 Elektrische Energie 389

10.7.2.8 Krankheit, Verletzung 390

10.7.3 Gefährlicher Einsatz, Einsatzgrundsatz 391

Register 393 
